\title{
A self-sustained traversable scale-dependent wormhole
}

\author{
Ernesto Contreras ${ }^{a * \dagger}$ and Pedro Bargueño ${ }^{a \ddagger}$ \\ ${ }^{a}$ Departamento de Física, Universidad de los Andes, \\ Apartado Aéreo 4976, Bogotá, Distrito Capital, Colombia
}

\begin{abstract}
A self-sustained traversable wormhole is obtained as a vacuum solution of a scale-dependent gravitational theory. Comparison with other approaches towards wormhole self-sustainability are presented, with emphasis on the running of the gravitational coupling and on a possible effective description of gravity near the Planck scale.
\end{abstract}

\section{INTRODUCTION}

Wormholes are bridges between different universes or different parts of the same universe. They were first recognized by Flamm, who found that the Schwarzschild solution of can be thought as representing a wormhole [1]. Later, Einstein and Rosen [2] developed a model for an elementary particle consisting in a throat connecting two sheets. After the "mass without mass"-like elaborations of Wheeler and Misner in their Geometrodynamics [3], the field experimented a renaissance due to the work of Morris and Thorne [4, 5]. In the last 20 years, the book by Visser [6] has been considered as an appropriate and authoritative reference in the field. Very recently, Lobo 7] has also authoritatively updated on the state-of-theart on wormhole physics. Therefore, after having a look at this timeline, one can conclude that we are living a new renaissance on wormholes and related physics.

Let us focus our interest in traversable wormholes. As it is well known, the problem with them is that they violate the classical energy conditions, serving primarily as useful probes of the foundations of General Relativity. The kind of matter which allows traversable wormholes is called exotic. As a consequence, quantum effects must be considered in order to solve the traversability problem. As the full theory of quantum gravity is still lacking, many different works have been devoted to get some insight into the underlying physics (for an incomplete list check [8 23] and for a review see [24]). Despite the fact that in those works the authors discuss different aspects of quantum gravity, most of them have the common feature that the resulting effective gravitational action acquires a scale-dependence. This behaviour is observed through the couplings of the effective action: they change from fixed values to scale-dependent quantities, i.e. $\left\{G_{0}, \Lambda_{0}\right\} \mapsto\left\{G_{k}, \Lambda_{k}\right\}$, where $G_{0}$ is Newton's coupling and $\Lambda_{0}$ is the cosmological coupling. Indeed, there is some evidence which supports that this scaling behaviour is consistent with Weinberg's Asymptotic Safety program 25 32. This effective action which appears when running couplings are assumed has been

\footnotetext{
* On leave from Universidad Central de Venezuela

$\dagger$ †j.contreras@uniandes.edu.co

${ }^{\ddagger}$ p.bargueno@uniandes.edu.co
}

studied in three-dimensional space-times in the context of black hole physics 33 36 as well as in four dimensions 37 39]. In these works, the corresponding scaledependent couplings take into account a quantum effect in the sense that this approach admits corrections to the classical black hole backgrounds.

From the point of view of Semiclassical General Relativity, self-consistent solutions to the semiclassical Einstein's equations corresponding to a Lorentzian wormhole coupled with a quantum scalar field have been considered by Hochberg et al. [40] and by Khusnutdinov and Sushkov [41]. Regarding self-sustainability, Garattini fixed the attention on wormholes which are totally supported by their own quantum fluctuations (see the original works [42, 43] and also [7] and references therein for a recent account of these kind of wormholes). By studying the one-loop contribution of the gravitons to the total energy, which is quite similar to computing the Casimir energy on a fixed background, he found a self-consistent source for a traversable wormhole [42]. An important feature of this self-sustainability lies in the fact that a renormalized energy-dependent Newton's gravitational constants appears as a consequence of considering effective Einstein's equations coming from the fluctuations of the Einstein tensor. Therefore, in this sense, an effective action description of self-sustaina ble wormholes should be possible. This description, within a scale-dependent gravitational setting, is the purpose of the present work.

The work is organized as follows: In Sect. III we give a brief review on traversable wormholes in Einstein's gravity. Section III summarizes the gravitational scaledependent setting which is employed in Sect. IV to obtain a self-sustained wormhole with the Schwarzschild spatial part of the metric. Finally, discussion and concluding remarks are given in Sects. V] and VI respectively.

\section{TRAVERSABLES WORMHOLES IN EINSTEIN'S GRAVITY}

Let us consider a Morris-Thorne wormhole [4], which is one of the simplest traversable wormholes. It can be described by a static and spherically symmetric line element 
given by

$$
d s^{2}=-e^{2 A(r)} d t^{2}+\frac{d r^{2}}{1-\frac{B(r)}{r}}+r^{2} d \Omega^{2} .
$$

For a static observer, the only nonzero components of the stress-energy tensor are

$$
\begin{aligned}
& T_{t}^{t}=\rho(r) \\
& T_{r}^{r}=-\tau(r) \\
& T_{\theta}^{\theta}=T_{\phi}^{\phi}=P(r),
\end{aligned}
$$

where $\rho(r)$ is the total density of mass-energy, $\tau(r)$ is the tension per unit of area in the radial direction and $P(r)$ is the pressure in lateral directions.

With the above parametrization of the line element and the choice (2) for the matter content, the Einstein's field equations lead to

$$
\begin{aligned}
\rho & =\frac{B^{\prime}}{8 \pi r^{2}} \\
\tau & =\frac{B / r-2(r-B) A^{\prime}}{8 \pi r^{2}} \\
P & =\frac{r}{2}\left((\rho-\tau) A^{\prime}-\tau^{\prime}\right)-\tau .
\end{aligned}
$$

The former equations suggest that, for a suitable choice of the functions $A(r)$ and $B(r)$, we can obtain the matter contain for our wormhole.

However, the functions $A(r)$ and $B(r)$ are not arbitrary but they must fulfill some constraints in order to obtain a traversable wormhole. For example, if there is not cutoff in the stress-energy we must demand that [4, 5]

$$
\begin{aligned}
\frac{B}{r} & \rightarrow 0 \\
A(r) & \rightarrow 0,
\end{aligned}
$$

as $r \rightarrow \infty$. Furthermore, the requirement that a traversa ble wormhole does not possess any horizon corresponds to demand $A(r)$ to be finite everywhere.

As an example, we will briefly comment on two types of traversable wormholes of ultrastatic [69] type.

First, the prototype of traversable wormhole, which is the Ellis-Bronnikov one 44-46]. This wormhole has a shape function given by $B(r)=r_{0}^{2} / r$ (with $r_{0}$ constant) and $A(r)=0$. Note that, although these wormholes were thought to be unstable [47 50], rotation might possibly stabilize them [51]. Even more, Bronnikov et al. have shown [52] that a perfect fluid with negative density and a source-free radial electric or magnetic field (for a certain class of fluid equations of state) allows linear stability for the Ellis-Bronnikov solution under both spherically symmetric perturbations and axial perturbations of arbitrary multipolarity (see also Bronnikov's study on Chapter 7 of [7]). Very recently, and in analogy with black holes [53], uniqueness theorems for the Ellis-Bronnikov wormhole supported by a phantom scalar field has been proven both in four [54] and in higher-dimensional cases [55]. Concerning the study of gravitational lensing of wormholes, due to their astrophysical importance, the deflection of light for Ellis-Bronnikov wormholes was initially computed in [56]. Other authors have extended the study of these kind of signatures both in non-rotating [57 63] and rotating Ellis-Bronnikov wormholes [64].

Second, let us consider a wormhole with $A(r)=0$ and $B(r)=r_{0}$. As pointed out by Morris and Thorne [4], the parameter $\xi=\frac{\tau}{\rho}-1$ quantifies the amount of exotic material needed to sustain the wormhole. In this particular case, although the exotic material decays rapidly with radius, $\xi$ is positive and huge. In this sense, the authors of Ref. [4] point out that this situation, which implies the use of exotic material throughout all the wormhole, is extremely unpleasing. Given this unpleasant feature of these wormholes when interpreted whithin General Relativity, in this work we will show that they are an exact vacuum solution of a particular scale-dependent gravity. Therefore, no exotic matter but a modified gravitational theory is implemented in order to obtain a self-sustained wormhole solution of this type.

\section{SCALE-DEPENDENT GRAVITY}

As commented in the introduction, one possible way of introducing an effective gravitational theory beyond General Relativity is by promoting both the Newton and the comological constants to scale-dependent quantities. In the following, the scale-setting presented will follow closely the spirit and concept of Ref. [39].

The scale-dependent Einstein-Hilbert effective action reads

$$
\Gamma\left[g_{\mu \nu}, k\right]=\int \mathrm{d}^{4} x \sqrt{-g}\left[\frac{1}{16 \pi G_{k}}\left(R-2 \Lambda_{k}\right)+\mathcal{L}_{k}^{\mathrm{M}}\right],
$$

where $G_{k}$ and $\Lambda_{k}$ stand for the scale-dependent gravitational and cosmological coupling, respectively, and $\mathcal{L}_{k}^{\mathrm{M}}$ is the Lagrangian density for the matter content.

After performing variations with respect to the metric field $g_{\mu \nu}$, we obtain the modified Einstein's field equations

$$
G_{\mu \nu}+g_{\mu \nu} \Lambda_{k}=8 \pi G_{k} T_{\mu \nu}^{e f f},
$$

where $T_{\mu \nu}^{e f f}$ is the effective energy-momentum tensor, defined as

$$
T_{\mu \nu}^{e f f}:=\left(T_{k}^{\mathrm{M}}\right)_{\mu \nu}-\frac{1}{8 \pi G_{k}} \Delta t_{\mu \nu} .
$$

In Eq. (10), $\left(T_{k}^{\mathrm{M}}\right)_{\mu \nu}$ is the matter energy-momentum tensor and $\Delta t_{\mu \nu}$ is given by

$$
\Delta t_{\mu \nu}=G_{k}\left(g_{\mu \nu} \square-\nabla_{\mu} \nabla_{\nu}\right) G_{k}^{-1} .
$$

As discussed previously in Ref. 34], the renormalization scale $k$ is not constant anymore. Therefore, the 
stress energy tensor is likely not conserved. This kind of problem has been considered in the context of renormalization group improvement of black holes in asymptotic safety scenarios (see, for instance [65 68] and references therein).

One can circumvent this problem by applying the variational scale-setting procedure described in Ref. [39], where the Eqs. (9) are complemented by an equation obtained performing variations with respect to the scalefield, $k(x)$ :

$$
\frac{\mathrm{d}}{\mathrm{d} k} \Gamma\left[g_{\mu \nu}, k\right]=0
$$

However, if the precise beta functions of the problem are not known, Eqs. (8) and (12) do not provide enough information in order to find both $g_{\mu \nu}(x)$ and $k(x)$. One can solve this problem by considering that the couplings $\left\{G_{k}, \Lambda_{k}\right\}$ depend explicitly on space-time coordinates, a dependence which is inherited from the space-time dependence of $k(x)$ [33 35, 37, 39]. Promoting Newton's coupling to a space-dependent field, $G(x)$, and finding wormhole solutions for this modified theory, is the purpose of the following section. Note that this scaledependent gravity corresponds to an effective BransDicke theory but without a kinetic term. In this sense, $G(x)$ does not have dynamics.

\section{SELF-SUSTAINED SCALE-DEPENDENT SOLUTION}

The modified vacuum Einstein's equations without cosmological term are given by $S_{\mu \nu}=G_{\mu \nu}+\Delta t_{\mu \nu}=0$, where

$$
\begin{aligned}
S_{t}^{t} & =-2 G^{2}(r) B^{\prime}(r)+4 r(r-B(r))\left(G^{\prime}(r)\right)^{2}+ \\
& +G(r)\left(3 B(r)+r\left(-4+B^{\prime}(r)\right)\right) G^{\prime}(r) \\
& \left.+2 r(-r+B(r)) G^{\prime \prime}(r)\right) \\
S_{r}^{r} & =G(r)\left(-B(r)+2 r(r-B(r)) A^{\prime}(r)\right) \\
& +r(-r+B(r))\left(2+r A^{\prime}(r)\right) G^{\prime}(r) \\
S_{\theta}^{\theta} & =S_{\phi}^{\phi}=B(r)\left(-4 r^{2} G^{\prime}(r)^{2}-G^{2}(r)\left(-1+r\left(A^{\prime}(r)\right.\right.\right. \\
& \left.\left.+2 r\left(A^{\prime}(r)\right)^{2}+2 r A^{\prime \prime}(r)\right)\right)+r G^{\prime}(r)\left(1+2 r A^{\prime}(r)\right) \times \\
\times & \left.\left.G^{\prime}(r)+2 r G^{\prime \prime}(r)\right)\right) r\left(4 r^{2}\left(G^{\prime}(r)\right)^{2}+G^{2}(r)((1+\right. \\
& \left.\left.\quad r A^{\prime}(r)\right)\left(2 r A^{\prime}(r)-B^{\prime}(r)\right)+2 r^{2} A^{\prime \prime}(r)\right)+r G(r) \times \\
\times & \left.\left(\left(-2-2 r A^{\prime}(r)+B^{\prime}(r)\right) G^{\prime}(r)-2 r G^{\prime \prime}(r)\right)\right)(15)
\end{aligned}
$$

Note that the equations are highly coupled. However, the following protocole can be implemented in order to look for some solutions. First, solve for $G^{\prime}(r)$ from Eq. (14). Second, substitute $G^{\prime}(r)$ in Eq. (13) and then solve for $G^{\prime \prime}(r)$. With these algebraic identities, Eq. (15) results in

$$
\begin{aligned}
& A^{\prime}(r)\left(-B(r)\left(6+r A^{\prime}(r)\left(1+r A^{\prime}(r)\right)\right)\right. \\
+ & \left.r\left(r A^{\prime}(r)\left(2+r A^{\prime}(r)-B^{\prime}(r)\right)-2\left(-8+B^{\prime}(r)\right)\right)\right) \\
+ & 8 B^{\prime}(r)+2 r(r-B(r))\left(4+r A^{\prime}(r)\right) A^{\prime \prime}(r)=0 .
\end{aligned}
$$

Surprisingly, Eq. (16) does not contain $G(r)$. Even more, one possible solution is given, by inspection, by

$$
\begin{aligned}
& A(r)=A_{0} \\
& B(r)=B_{0} .
\end{aligned}
$$

Note that, given this choice for the metric, all the equations (where $G(r)$ is the only unknown) can be consistently solved leading to

$$
G(r)=\frac{G_{0}}{\sqrt{1-\frac{B_{0}}{r}}},
$$

where $G_{0}$ is the classical Newton's constant. It is worth noticing that, as the redshift is constant $\left(A_{0}\right)$, the radial tidal acceleration felt by an observer trying to traverse the wormhole is zero. On the contrary, the transversal tidal acceleration essentially depends on the velocity with which the observer traverses the wormhole [4, 6, 7].

\section{DISCUSSION}

At this point, a number of comments are in order. First, note that the spatial part of the obtained wormhole is similar to that of a Schwarzschild wormhole (the Schwarzschild redshift is somehow incorporated in the effective $G(r)$ ). Second, in the context of scale-dependent gravity, the wormhole throat, $B_{0}$, can be interpreted as the so-called running parameter, which controls the strength of the scale-dependence [33 35, 37, 39]. In other words, when the running parameter is turned off, $B_{0} \rightarrow$ 0 , the classical solution is recovered, and $G(r) \rightarrow G_{0}$. Even more, this limit corresponds to Minkowski spacetime, as can be easily checked. In this sense, the solution here presented can be considered to be self-sustained by a scale-dependent gravitational theory where the effective Newton's constant is given by Eq. (19). Third, as in general the scale-dependent effects are assumed to be weak [37], it is reasonable to treat the running parameter, which we recall is encoded in $B_{0}$, as small with respect to the other scales entering the problem. Therefore, the effects of the running of $G(r)$ [70] are expected to be noticeable only near the throat. Specifically, as $\left[B_{0}\right]=L$ and $\left[G_{0}\right]=[L]^{1 / 2}$ when $c=\hbar=1$, we get that $B_{0}<\sqrt{G_{0}}=l_{p}$. Then, provided scale-dependent 
gravity can be considered as an effective model for quantum gravity in some sense, the (trans)-planckian bound obtained for $B_{0}$ is consistent and, even more, it is in agreement with [41 43].

Within this interpretation, no violation of energy conditions appears, since we are dealing with a vacuum spacetime, but a modified gravity emerges. In fact, as pointed out in Ref. [7]: "in the context of modified theories of gravity, it is shown that the higher-order curvature terms, interpreted as a gravitational fluid, can effectively sustain wormhole geometries, while the matter threading the wormhole can be imposed to satisfy the energy conditions". In our case, the matter content which Ref. 7] refers to is the vacuum and the new gravity is not given by higher-order curvature terms but by the scaledependence. Therefore, scale-dependent gravity provides a possible realization of the previous claim.

Concerning the self-sustainability of the wormhole note that, one one hand, in the approach of Refs. [42, 43], the effective Einstein's equations are given by

$$
G_{\mu \nu}=-\left\langle\Delta G_{\mu \nu}\left(\bar{g}_{\mu \nu}, h_{\mu \nu}\right)\right\rangle^{\mathrm{ren}}
$$

where $-\left\langle\Delta G_{\mu \nu}\left(\bar{g}_{\mu \nu}, h_{\mu \nu}\right)\right\rangle^{\text {ren }}$ is an effective energy-mom entum tensor which appears as a consequence of a oneloop renormalization procedure over a fixed wormhole background given by $g_{\mu \nu}\left(\bar{g}_{\mu \nu}=g_{\mu \nu}+h_{\mu \nu}\right)$. Moreover, given the fact that an arbitrary mass scale, $\mu$, emerges unavoidably in any regularization scheme, a scale-dependent running gravitational coupling appears. The specific running obtained in Ref. [42] reads

$$
G(\mu)=\frac{G_{0}}{1+K G_{0} \ln \left(\mu / \mu_{0}\right)},
$$

where $K$ is a constant related to the background geometry and $\mu_{0}$ is the normalization point. On the other hand, within our approach, the effective Einstein's equations are given by

$$
G_{\mu \nu}=-\Delta t_{\mu \nu}
$$

where the effective energy-momentum tensor appears when the scale-dependence can not be avoided anymore. Therefore, one can conclude that the scale-depen dence of the gravitational coupling provides an effective mechanism for the inclussion of quantum effects in the context of wormholes. In this sense, the obtained solution can be also taken to be self-sustainable, but this time due to the effect of the running of the Newton's gravitational coupling.

\section{CONCLUDING REMARKS}

In this work we have constructed the first wormhole solution in the context of scale-dependent gravity. Interestingly, the obtained geometry is a vacuum solution of the modified Einstein's equations and, therefore, no violations of the energy conditions appear. The width of the wormhole's throat has been shown to correspond to the running parameter, which measures deviations from General Relativity. Even more, this parameter controls the running of the Newton's coupling, which appears to be redshifted instead being constant as in the usual case. We have noted that this wormhole is self-sustained in the sense that the obtained effective gravity is the only responsible of its sustainability. In this sense, the model here presented can be thought as an effective description of previously considered self-sustained wormholes, which is confirmed by their (trans)-planckian size. Therefore, following [42, 43], we conclude that the obtained traversability has to be regarded as in "principle" rather than in "practice". Finally, in order to propose some astrophysical signatures of the wormhole here presented, a study of its stability is mandatory. We leave this and other topics for a future work.

\section{ACKNOWLEDGEMENTS}

The author P. B. was supported by the Faculty of Science and Vicerrectoría de Investigaciones of Universidad de los Andes, Bogotá, Colombia. P. B. dedicates this work to Anaís Dorta-Urra and to Lucía and Inés BargueñoDorta.
[1] L. Flamm, Physik Z. 17, 448 (1916).

[2] A. Einstein and N. Rosen, Phys Rev. 48, 73 (1935).

[3] C. W. Misner and J. A. Wheeler, Annals Phys. 2, 525 (1957).

[4] M. S. Morris and K.S. Thorne, Am. J. Phys. 56, 395 (1988).

[5] M. S. Morris and K.S. Thorne and U. Yurtsever, Phys. Rev. Lett. 61, 1446 (1988).

[6] M. Visser, Lorentzian wormholes: From Einstein to Hawking, AIP Press, NY (1996).

[7] F. S. N. (editor), Wormholes, warp drives and energy conditions, Springer (2017).

[8] S. Deser and B. Zumino, Phys. Lett. 62B, 335 (1976).

[9] C. Rovelli, Living Rev. Rel. 1, 1 (1998).

[10] L. Bombelli, J. Lee, D. Meyer and R. Sorkin, Phys. Rev. Lett. 59, 521 (1987).

[11] A. Ashtekar, New J. Phys. 7, 198 (2005).

[12] A. D. Sakharov, Sov. Phys. Dokl. 12, 1040 (1968) [Dokl. Akad. Nauk Ser. Fiz. 177, 70 (1967)] [Sov. Phys. Usp. 34, 394 (1991)] [Gen. Rel. Grav. 32, 365 (2000)].

[13] T. Jacobson, Phys. Rev. Lett. 75, 1260 (1995).

[14] E. P. Verlinde, JHEP 1104, 029 (2011). 
[15] M. Reuter, Phys. Rev. D 57, 971 (1998).

[16] D. F. Litim, Phys. Rev. Lett. 92, 201301 (2004).

[17] P. Horava, Phys. Rev. D 79, 084008 (2009).

[18] C. Charmousis, G. Niz, A. Padilla and P. M. Saffin, JHEP 0908, 070 (2009).

[19] A. Ashtekar, Phys. Rev. Lett. 46, 573 (1981).

[20] R. Penrose and W. Rindler, "Spinors And Space-time. Vol. 2: Spinor And Twistor Methods In Space-time Geometry".

[21] A. Connes, Commun. Math. Phys. 182, 155 (1996).

[22] P. Nicolini, Int. J. Mod. Phys. A 24, 1229 (2009).

[23] R. Gambini and J. Pullin, Phys. Rev. Lett. 94, 101302 (2005).

[24] C. Kiefer, Annalen Phys. 15, 129 (2005).

[25] S., and Israel, W., eds. Hawking, Cambridge, UK: Cambridge University Press (1979) pp 935.

[26] C. Wetterich, Phys. Lett. B 301, 90 (1993).

[27] D. Dou and R. Percacci, Class. Quant. Grav. 15, 3449 (1998).

[28] W. Souma, Prog. Theor. Phys. 102, 181 (1999).

[29] M. Reuter and F. Saueressig, Phys. Rev. D 65, 065016 (2002).

[30] P. Fischer and D. F. Litim, Phys. Lett. B 638, 497 (2006).

[31] R. Percacci, In Oriti, D. (ed.): Approaches to quantum gravity 111-128.

[32] D. F. Litim, arXiv:0810.3675 [hep-th].

[33] B. Koch, I. A. Reyes and Á. Rincón, Class. Quant. Grav. 33, no. 2, 225010 (2016).

[34] Á. Rincón, E. Contreras, P. Bargueño, B. Koch, G. Panotopoulos and A. Hernández-Arboleda, Eur. Phys. J. C 77, 494 (2017).

[35] Á. Rincón, B. Koch and I. Reyes, J. Phys. Conf. Ser. 831, no. 1, 012007 (2017).

[36] Á. Rincón and G. Panotopoulos, Phys. Rev. D (in press, 2018).

[37] B. Koch and P. Rioseco, Class. Quantum Grav. 33, 035002 (2016).

[38] E. Contreras, Á. Rincón, B. Koch and P. Bargueño, Int. J. Mod. Phys. D, https://doi.org/10.1142/S0218271818500323 (2018).

[39] B. Koch, P. Rioseco and C. Contreras, Phys. Rev. D 91, no. 2, 025009 (2015).

[40] D. Hochberg, A. Popov S. V. and Sushkov, Phys. Rev. Lett. 78, 2050 (1997).

[41] N. R. Khusnutdinov and S. V. Sushkov, Phys. Rev. D 65 084028 (2002).
[42] R. Garattini, Class. Quantum Grav. 22, 1105 (2005).

[43] R. Garattini, Class. Quantum Grav. 24, 1189 (2007).

[44] H. G. Ellis, J. Math. Phys. 14, 104 (1973).

[45] K. A. Bronnikov, Acta Physica Polonica B 4, 251 (1973).

[46] H. G. Ellis, Gen. Rel. Grav. 10, 105 (1979).

[47] H. A. Shinkai and S. A. Hayward, Phys. Rev. D. 66, 044005 (2002).

[48] J. A. Gonzalez, F. S. Guzman and O. Sarbach, Class. Quant. Grav. 26, 015010 (2009).

[49] J. A. Gonzalez, F. S. Guzman and O. Sarbach, Class. Quant. Grav. 26, 015011 (2009).

[50] T. Torii and H. Shinkai, Phys. Rev. D 88, 064027 (2013).

[51] T. Matos and D. Nunez, Class. Quant. Grav. 23, 4485 (2006).

[52] K. A. Bronnikov, L. N. Lipatova, I. D. Novikov and A. A. Shatskiy, Gravitation and Cosmology, 19, 269 (2013).

[53] M. Heusler, Stationary Black Holes: Uniqueness and Beyond, Springer (1998).

[54] S. Yazadjiev, Phys. Rev. D 96, 044045 (2017).

[55] M. Rogatko, Phys. Rev. D 97, 024001 (2018).

[56] L. Chetouani and G. Clement, Gen. Rel. Gravit. 16, 111 (1984).

[57] F. Abe, Astrophys. J. 725, 787 (2010).

[58] Y. Toki, T. Kitamura, H. Asada and F. Abe, Astrophys. J. 740, 121 (2011).

[59] R. Takahashi and H. Asada, Astrophys. J. 768, L16 (2013).

[60] M. A. Cardenas-Avendano, C. Bambi, B. Kleihaus and J. Kunz, Phys. Rev. D. 94, 024036 (2016).

[61] N. Tsukamoto, Phys. Rev. D 94, 124001 (2016).

[62] N. Tsukamoto and T. Harada, Phys. Rev. D 95, 024030 (2017).

[63] N. Tsukamoto, Phys. Rev. D 95, 084021 (2017).

[64] K. Jusufi and A. Övgün, Phys. Rev. D 97, 024042 (2018).

[65] A. Bonanno and M. Reuter, Phys. Rev. D 62, 043008 (2000).

[66] A. Bonanno and M. Reuter, Phys. Rev. D 73, 083005 (2006).

[67] M. Reuter and E. Tuiran, Phys. Rev. D 83, 044041 (2011).

[68] B. Koch and F. Saueressig, Int. J. Mod. Phys. A 29, no. 8, 1430011 (2014).

[69] A spacetime is ultrastatic if can be written in some coordinate system as $g=-d t^{2}+g_{i j} d x^{i} d x^{j}$

[70] $G(r)$ can be considered to be an effective beta function in the spirit of [37]. 